\title{
An inverse coefficient problem for a quasilinear parabolic equation with nonlocal boundary conditions
}

Fatma Kanca ${ }^{1 *}$ and Irem Baglan ${ }^{2}$

"Correspondence:

fatma.kanca@khas.edu.tr

'Department of Management

Information Systems, Kadir Has University, Istanbul, 34083, Turkey

Full list of author information is

available at the end of the article

\begin{abstract}
In this paper the inverse problem of finding the time-dependent coefficient of heat capacity together with the nonlocal boundary conditions is considered. Under some natural regularity and consistency conditions on the input data, the existence, uniqueness and continuous dependence upon the data of the solution are shown. Some considerations on the numerical solution for this inverse problem are presented with an example.
\end{abstract}

\section{Introduction}

Denote the domain $D$ by

$$
D:=\{0<x<1,0<t<T\} .
$$

Consider the equation

$$
u_{t}=u_{x x}-p(t) u+f(x, t, u)
$$

with the initial condition

$$
u(x, 0)=\varphi(x), \quad x \in[0,1]
$$

the nonlocal boundary condition

$$
u(0, t)=u(1, t), \quad u_{x}(1, t)=0, \quad t \in[0, T],
$$

and the overdetermination data

$$
u_{x}(0, t)=g(t), \quad t \in[0, T]
$$

for a quasilinear parabolic equation with the nonlinear source term $f=f(x, t, u)$.

The functions $\varphi(x)$ and $f(x, t, u)$ are given functions on $[0,1]$ and $\bar{D} \times(-\infty, \infty)$, respectively.

The problem of finding the pair $\{p(t), u(x, t)\}$ in (1)-(4) will be called an inverse problem.

O2013 Kanca and Baglan; licensee Springer. This is an Open Access article distributed under the terms of the Creative Commons Attribution License (http://creativecommons.org/licenses/by/2.0), which permits unrestricted use, distribution, and reproduction in any medium, provided the original work is properly cited. 
Definition 1 The pair $\{p(t), u(x, t)\}$ from the class $C[0, T] \times\left(C^{2,1}(D) \cap C^{1,0}(\bar{D})\right)$, for which conditions (1)-(4) are satisfied and $p(t) \geq 0$ on the interval $[0, T]$, is called the classical solution of inverse problem (1)-(4).

The problem of identification of a coefficient in a nonlinear parabolic equation is an interesting problem for many scientists [1-3]. Inverse problems for parabolic equations with nonlocal boundary conditions are investigated in [4-6]. This kind of conditions arise from many important applications in heat transfer, life sciences, etc. In [7], also the nature of (3) type boundary conditions is demonstrated.

In [1] the boundary conditions are local, the solution is obtained locally and the authors obtained the solution in Holder classes using iteration method. In [5] the boundary condition is nonlocal but the problem is linear and the existence and the uniqueness of the classical solution is obtained locally using a fixed point theorem. In this paper, the existence and uniqueness of the classical solution is obtained locally using the iteration method.

The paper is organized as follows. In Section 2, the existence and uniqueness of the solution of inverse problem (1)-(4) is proved by using the Fourier method and the iteration method. In Section 3, the continuous dependence upon the data of the inverse problem is shown. In Section 4, the numerical procedure for the solution of the inverse problem is given.

\section{Existence and uniqueness of the solution of the inverse problem}

Consider the following system of functions on the interval $[0,1]$ :

$$
\begin{array}{lll}
X_{0}(x)=2, & X_{2 k-1}(x)=4 \cos (2 \pi k x), & X_{2 k}(x)=4(1-x) \sin (2 \pi k x), \quad k=1,2, \ldots, \\
Y_{0}(x)=x, & Y_{2 k-1}(x)=x \cos (2 \pi k x), & Y_{2 k}(x)=\sin (2 \pi k x), \quad k=1,2, \ldots .
\end{array}
$$

The systems of these functions arise in [8] for the solution of a nonlocal boundary value problem in heat conduction. It is easy to verify that the system of functions $X_{k}(x)$ and $Y_{k}(x), k=0,1,2, \ldots$, is biorthonormal on $[0,1]$. They are also Riesz bases in $L_{2}[0,1]$ (see $[5,6])$.

The main result on the existence and uniqueness of the solution of inverse problem (1)(4) is presented as follows.

We have the following assumptions on the data of problem (1)-(4):

(A1) $g(t) \in C^{1}[0, T], g(t)<0, g^{\prime}(t) \geq 0$;

(A2) $\varphi(x) \in C^{3}[0,1]$

(1) $\varphi(0)=\varphi(1), \varphi^{\prime}(1)=0, \varphi^{\prime \prime}(0)=\varphi^{\prime \prime}(1)$,

(2) $\varphi_{2 k} \geq 0, k=1,2, \ldots$;

(A3) Let the function $f(x, t, u)$ be continuous with respect to all arguments in $\bar{D} \times(-\infty, \infty)$ and satisfy the following conditions:

(1)

$$
\left|f^{(n)}(x, t, u)-f^{(n)}(x, t, \tilde{u})\right| \leq b(t, x)|u-\tilde{u}|, \quad n=0,1,2,
$$

where $b(x, t) \in L_{2}(D), b(x, t) \geq 0$,

(2) $f(x, t, u) \in C^{3}[0,1], t \in[0, T]$, 
(3) $\left.f(x, t, u)\right|_{x=0}=\left.f(x, t, u)\right|_{x=1},\left.f_{x}(x, t, u)\right|_{x=1}=0,\left.f_{x x}(x, t, u)\right|_{x=0}=\left.f_{x x}(x, t, u)\right|_{x=1}$,

(4) $f_{2 k}(t) \geq 0, f_{0}(t)+\sum_{k=1}^{\infty}(2 \pi k)^{2}\left(\varphi_{2 k}+\int_{0}^{T} f_{2 k}(\tau) d \tau\right) \leq g^{\prime}(t), \forall t \in[0, T]$, where

$$
\varphi_{k}=\int_{0}^{1} \varphi(x) Y_{k}(x) d x, \quad f_{k}(t)=\int_{0}^{1} f(x, t, u) Y_{k}(x) d x, \quad k=0,1,2, \ldots
$$

By applying the standard procedure of the Fourier method, we obtain the following representation for the solution of (1)-(3) for arbitrary $p(t) \in C[0, T]$ :

$$
\begin{aligned}
& u(x, t)=\left[\varphi_{0} e^{-\int_{0}^{t} p(s) d s}+\int_{0}^{t} \int_{0}^{1} f(\xi, \tau, u) \xi e^{-\int_{\tau}^{t} p(s) d s} d \xi d \tau\right] X_{0}(x) \\
& +\sum_{k=1}^{\infty} X_{2 k}(x)\left[\varphi_{2 k} e^{-(2 \pi k)^{2} t-\int_{0}^{t} p(s) d s}\right. \\
& \left.+\int_{0}^{t} \int_{0}^{1} f(\xi, \tau, u) \sin 2 \pi k \xi e^{-(2 \pi k)^{2}(t-\tau)-\int_{\tau}^{t} p(s) d s} d \xi d \tau\right] \\
& +\sum_{k=1}^{\infty} X_{2 k-1}(x)\left[\left(\varphi_{2 k-1}-4 \pi k t \varphi_{2 k}\right) e^{-(2 \pi k)^{2} t-\int_{0}^{t} p(s) d s}\right] \\
& +\sum_{k=1}^{\infty} X_{2 k-1}(x)\left[\int_{0}^{t} \int_{0}^{1} f(\xi, \tau, u) \xi \cos 2 k \xi e^{-(2 \pi k)^{2}(t-\tau)-\int_{\tau}^{t} p(s) d s} d \xi d \tau\right] \\
& -\sum_{k=1}^{\infty} X_{2 k-1}(x) \\
& \times\left[4 \pi k \int_{0}^{t} \int_{0}^{1} f(\xi, \tau, u)(t-\tau) \sin 2 \pi k \xi e^{-(2 \pi k)^{2}(t-\tau)-\int_{\tau}^{t} p(s) d s} d \xi d \tau\right], \\
& u_{0}(t)=\varphi_{0} e^{-\int_{0}^{t} p(s) d s}+\int_{0}^{t} \int_{0}^{1} f(\xi, \tau, u) \xi e^{-\int_{\tau}^{t} p(s) d s} d \xi d \tau, \\
& u_{2 k}(t)=\varphi_{2 k} e^{-(2 \pi k)^{2} t-\int_{0}^{t} p(s) d s}+\int_{0}^{t} \int_{0}^{1} f(\xi, \tau, u) \sin 2 \pi k \xi e^{-(2 \pi k)^{2}(t-\tau)-\int_{\tau}^{t} p(s) d s} d \xi d \tau, \\
& u_{2 k-1}(t)=\left(\varphi_{2 k-1}-4 \pi k t \varphi_{2 k}\right) e^{-(2 \pi k)^{2} t-\int_{0}^{t} p(s) d s} \\
& +\int_{0}^{t} \int_{0}^{1} f(\xi, \tau, u) \xi \cos 2 k \xi e^{-(2 \pi k)^{2}(t-\tau)-\int_{\tau}^{t} p(s) d s} d \xi d \tau \\
& -4 \pi k \int_{0}^{t} \int_{0}^{1} f(\xi, \tau, u)(t-\tau) \sin 2 \pi k \xi e^{-(2 \pi k)^{2}(t-\tau)-\int_{\tau}^{t} p(s) d s} d \xi d \tau .
\end{aligned}
$$

Under conditions (A1)-(A3), we obtain

$$
u_{t x}(0, t)=g^{\prime}(t), \quad 0 \leq t \leq T .
$$

Equations (5) and (6) yield

$$
\begin{aligned}
p(t)= & \frac{1}{g(t)}\left[-g^{\prime}(t)+\sum_{k=1}^{\infty}\left(8 \pi k f_{2 k}-4(2 \pi k)^{2} \varphi_{2 k} e^{-(2 \pi k)^{2} t-\int_{0}^{t} p(s) d s}\right)\right] \\
& -\frac{1}{g(t)} \sum_{k=1}^{\infty} 4(2 \pi k)^{2} \int_{0}^{t} f_{2 k}(t) e^{-(2 \pi k)^{2}(t-\tau)-\int_{\tau}^{t} p(s) d s} d \tau .
\end{aligned}
$$


Definition 2 Denote the set $\{u(t)\}=\left\{u_{0}(t), u_{2 k}(t), u_{2 k-1}(t), k=1, \ldots, n\right\}$ of continuous on $[0, T]$ functions satisfying the condition $2 \max _{0 \leq t \leq T}\left|u_{0}(t)\right|+4 \sum_{k=1}^{\infty}\left(\max _{0 \leq t \leq T}\left|u_{2 k}(t)\right|+\right.$ $\left.\max _{0 \leq t \leq T}\left|u_{2 k-1}(t)\right|\right)<\infty$ by B. Let $\|u(t)\|=2 \max _{0 \leq t \leq T}\left|u_{0}(t)\right|+4 \sum_{k=1}^{\infty}\left(\max _{0 \leq t \leq T}\left|u_{2 k}(t)\right|+\right.$ $\left.\max _{0 \leq t \leq T}\left|u_{2 k-1}(t)\right|\right)$ be the norm in $\mathbf{B}$. It can be shown that $\mathbf{B}$ is the Banach space.

Theorem 3 Let assumptions (A1)-(A3) be satisfied. Then inverse problem (1)-(4) has a unique solution for small $T$.

Proof An iteration for (5) is defined as follows:

$$
\begin{aligned}
u_{0}^{(N+1)}(t)= & u_{0}^{(0)}(t)+\int_{0}^{t} \int_{0}^{1} f(\xi, \tau, u) \xi e^{-\int_{\tau}^{t} p^{(N)}(s) d s} d \xi d \tau, \\
u_{2 k}^{(N+1)}(t)= & u_{2 k}^{(0)}(t)+\int_{0}^{t} \int_{0}^{1} f(\xi, \tau, u) \sin 2 \pi k \xi e^{-(2 \pi k)^{2}(t-\tau)-\int_{\tau}^{t} p^{(N)}(s) d s} d \xi d \tau, \\
u_{2 k-1}^{(N+1)}(t)= & u_{2 k-1}^{(0)}(t)+\int_{0}^{t} \int_{0}^{1} f(\xi, \tau, u) \xi \cos 2 k \xi e^{-(2 \pi k)^{2}(t-\tau)-\int_{\tau}^{t} p^{(N)}(s) d s} d \xi d \tau \\
& -4 \pi k \int_{0}^{t} \int_{0}^{1} f(\xi, \tau, u)(t-\tau) \sin 2 \pi k \xi e^{-(2 \pi k)^{2}(t-\tau)-\int_{\tau}^{t} p^{(N)}(s) d s} d \xi d \tau,
\end{aligned}
$$

where $N=0,1,2, \ldots$ and

$$
\begin{aligned}
& u_{0}^{(0)}(t)=\varphi_{0} e^{-\int_{0}^{t} p(s) d s}, \quad u_{2 k}^{(0)}(t)=\varphi_{2 k} e^{-(2 \pi k)^{2} t-\int_{0}^{t} p(s) d s}, \\
& u_{2 k-1}^{(0)}(t)=\left(\varphi_{2 k-1}-4 \pi k t \varphi_{2 k}\right) e^{-(2 \pi k)^{2} t-\int_{0}^{t} p(s) d s} .
\end{aligned}
$$

From the conditions of the theorem, we have $u^{(0)}(t) \in \mathbf{B}$, and let $p^{(0)}=0$.

Let us write $N=0$ in (8).

$$
u_{0}^{(1)}(t)=u_{0}^{(0)}(t)+\int_{0}^{t} \int_{0}^{1} f(\xi, \tau, u) \xi d \xi d \tau
$$

Adding and subtracting $\int_{0}^{t} \int_{0}^{1} f(\xi, \tau, 0) d \xi d \tau$ on both sides of the last equation, we obtain

$$
u_{0}^{(1)}(t)=u_{0}^{(0)}(t)+\int_{0}^{t} \int_{0}^{1}\left[f\left(\xi, \tau, u^{(0)}(\xi, \tau)\right)-f(\xi, \tau, 0)\right] d \xi d \tau+\int_{0}^{t} \int_{0}^{1} f(\xi, \tau, 0) d \xi d \tau
$$

Applying the Cauchy inequality and the Lipschitz condition to the last equation and taking the maximum of both sides of the last inequality yields the following:

$$
\begin{aligned}
& \max _{0 \leq t \leq T}\left|u_{0}^{(1)}(t)\right| \leq\left|\varphi_{0}\right|+\sqrt{T}\|b(x, t)\|_{L_{2}(D)}\left\|u^{(0)}(t)\right\|+\sqrt{T}\|f(x, t, 0)\|_{L_{2}(D)}, \\
& u_{2 k}^{(1)}(t)= \varphi_{2 k} e^{-(2 \pi k)^{2} t}+\int_{0}^{t} \int_{0}^{1}\left[f\left(\xi, \tau, u^{(0)}\right)-f(\xi, \tau, 0)\right] \sin 2 \pi k \xi e^{-(2 \pi k)^{2}(t-\tau)} d \xi d \tau \\
&+\int_{0}^{t} \int_{0}^{1} f(\xi, \tau, 0) \sin 2 \pi k \xi e^{-(2 \pi k)^{2}(t-\tau)} d \xi d \tau .
\end{aligned}
$$


Applying Cauchy's inequality, Hölder's inequality, Bessel's inequality, the Lipschitz condition and taking maximum of both sides of the last inequality yields the following:

$$
\begin{aligned}
& \sum_{k=1}^{\infty} \max _{0 \leq t \leq T}\left|u_{2 k}^{(1)}(t)\right| \\
& \quad \leq \sum_{k=1}^{\infty}\left|\varphi_{2 k}\right|+\frac{\sqrt{3}}{3}\|b(x, t)\|_{L_{2}(D)}\left\|u^{(0)}(t)\right\|+\frac{\sqrt{3}}{3}\|f(x, t, 0)\|_{L_{2}(D)} .
\end{aligned}
$$

Applying the same estimations, we obtain

$$
\begin{aligned}
& \sum_{k=1}^{\infty} \max _{0 \leq t \leq T}\left|u_{2 k-1}^{(1)}(t)\right| \\
& \quad \leq \sum_{k=1}^{\infty}\left|\varphi_{2 k-1}\right|+\frac{2 \sqrt{6} T}{3} \sum_{k=1}^{\infty}\left|\varphi_{2 k}^{\prime \prime}\right| \\
& \quad+\left(\frac{\sqrt{3}}{3}+2 \sqrt{2}|T|\right)\|b(x, t)\|_{L_{2}(D)}\left\|u^{(0)}(t)\right\|+\left(\frac{\sqrt{3}}{3}+2 \sqrt{2}|T|\right)\|f(x, t, 0)\|_{L_{2}(D)^{\circ}}
\end{aligned}
$$

Finally, we have the following inequality:

$$
\begin{aligned}
\left\|u^{(1)}(t)\right\|_{B}= & 2 \max _{0 \leq t \leq T}\left|u_{0}^{(1)}(t)\right|+4 \sum_{k=1}^{\infty}\left(\max _{0 \leq t \leq T}\left|u_{2 k}^{(1)}(t)\right|+\max _{0 \leq t \leq T}\left|u_{2 k-1}^{(1)}(t)\right|\right) \\
\leq & \left|\varphi_{0}\right|+4 \sum_{k=1}^{\infty}\left(\left|\varphi_{2 k}\right|+\left|\varphi_{2 k-1}\right|\right)+\frac{2 \sqrt{6} T}{3} \sum_{k=1}^{\infty}\left|\varphi_{2 k}^{\prime \prime}\right| \\
& +\left(2 \sqrt{T}+\frac{2 \sqrt{3}}{3}+4 \sqrt{2}|T|\right)\left(\|b(x, t)\|_{L_{2}(D)}\left\|u^{(0)}(t)\right\|_{B}\right) \\
& +\left(2 \sqrt{T}+\frac{2 \sqrt{3}}{3}+4 \sqrt{2}|T|\right)\|f(x, t, 0)\|_{L_{2}(D)} .
\end{aligned}
$$

Hence $u^{(1)}(t) \in \mathbf{B}$. In the same way, for a general value of $N$, we have

$$
\begin{aligned}
\left\|u^{(N)}(t)\right\|_{B}= & 2 \max _{0 \leq t \leq T}\left|u_{0}^{(N)}(t)\right|+4 \sum_{k=1}^{\infty}\left(\max _{0 \leq t \leq T}\left|u_{2 k}^{(N)}(t)\right|+\max _{0 \leq t \leq T}\left|u_{2 k-1}^{(N)}(t)\right|\right) \\
\leq & \left|\varphi_{0}\right|+4 \sum_{k=1}^{\infty}\left(\left|\varphi_{c k}\right|+\left|\varphi_{s k}\right|\right)+\frac{2 \sqrt{6} T}{3} \sum_{k=1}^{\infty}\left|\varphi_{2 k}^{\prime \prime}\right| \\
& +\left(2 \sqrt{T}+\frac{2 \sqrt{3}}{3}+4 \sqrt{2}|T|\right)\left(\|b(x, t)\|_{L_{2}(D)}\left\|u^{(N-1)}(t)\right\|_{B}\right) \\
& +\left(2 \sqrt{T}+\frac{2 \sqrt{3}}{3}+4 \sqrt{2}|T|\right)\|f(x, t, 0)\|_{L_{2}(D)} .
\end{aligned}
$$

From $u^{(N-1)}(t) \in \mathbf{B}$ we deduce that $u^{(N)}(t) \in \mathbf{B}$,

$$
\{u(t)\}=\left\{u_{0}(t), u_{2 k}(t), u_{2 k-1}(t), k=1,2, \ldots\right\} \in B .
$$


An iteration for (7) is defined as follows:

$$
\begin{aligned}
p^{(N+1)}(t)= & \frac{1}{g(t)}\left[-g^{\prime}(t)+\sum_{k=1}^{\infty}\left(8 \pi k \int_{0}^{1} f\left(\xi, \tau, u^{(N)}\right) \sin 2 \pi k \xi d \xi\right.\right. \\
& \left.\left.-4(2 \pi k)^{2} \varphi_{2 k} e^{-(2 \pi k)^{2} t-\int_{0}^{t} p^{(N)}(s) d s}\right)\right] \\
& -\frac{1}{g(t)} \sum_{k=1}^{\infty} 4(2 \pi k)^{2} \int_{0}^{t} \int_{0}^{1} f\left(\xi, \tau, u^{(N)}\right) \sin 2 \pi k \xi e^{-(2 \pi k)^{2}(t-\tau)-\int_{\tau}^{t} p^{(N)}(s) d s} d \xi d \tau,
\end{aligned}
$$

where $N=0,1,2, \ldots$,

$$
\begin{aligned}
p^{(1)}(t)= & \frac{1}{g(t)}\left[-g^{\prime}(t)+\sum_{k=1}^{\infty}\left(8 \pi k \int_{0}^{1} f\left(\xi, \tau, u^{(0)}\right) \sin 2 \pi k \xi d \xi-4(2 \pi k)^{2} \varphi_{2 k} e^{-(2 \pi k)^{2} t}\right)\right] \\
& -\frac{1}{g(t)} \sum_{k=1}^{\infty} 4(2 \pi k)^{2} \int_{0}^{t} \int_{0}^{1} f\left(\xi, \tau, u^{(0)}\right) \sin 2 \pi k \xi e^{-(2 \pi k)^{2}(t-\tau)} d \xi d \tau .
\end{aligned}
$$

For convergence,

$$
\begin{aligned}
p^{(1)}(t)= & \frac{1}{g(t)}\left[-g^{\prime}(t)+\sum_{k=1}^{\infty} \frac{8 \pi k}{(2 \pi k)^{2}} \int_{0}^{1} f_{\xi \xi}\left(\xi, \tau, u^{(0)}\right) \sin 2 \pi k \xi d \xi\right. \\
& \left.-\sum_{k=1}^{\infty} \frac{4(2 \pi k)^{2}}{(2 \pi k)^{3}} \varphi_{2 k}^{\prime \prime \prime} e^{-(2 \pi k)^{2} t}\right] \\
& -\frac{1}{g(t)} \sum_{k=1}^{\infty} \frac{4(2 \pi k)^{2}}{(2 \pi k)^{2}} \int_{0}^{t} \int_{0}^{1} f_{\xi \xi}\left(\xi, \tau, u^{(0)}\right) \sin 2 \pi k \xi e^{-(2 \pi k)^{2}(t-\tau)} d \xi d \tau .
\end{aligned}
$$

Applying Cauchy's inequality, Hölder's inequality, Bessel's inequality, the Lipschitz condition and taking maximum of both sides of the last inequality yields the following:

$$
\begin{aligned}
\left|p^{(1)}(t)\right| \leq & \left|\frac{g^{\prime}(t)}{g(t)}\right|+\frac{\sqrt{6}}{3|g(t)|} \sum_{k=1}^{\infty}\left|\varphi_{2 k}^{\prime \prime \prime}\right| \\
& +\left(\frac{\sqrt{6}+\sqrt{3}}{3|g(t)|}\right)\|b(x, t)\|_{L_{2}(D)}\left\|u^{(0)}(t)\right\|_{B} \\
& +\left(\frac{\sqrt{6}+\sqrt{3}}{3|g(t)|}\right) M .
\end{aligned}
$$

Hence $p^{(1)}(t) \in \mathbf{B}$. In the same way, for a general value of $N$, we have

$$
\begin{aligned}
\left|p^{(N+1)}(t)\right| \leq & \left|\frac{g^{\prime}(t)}{g(t)}\right|+\frac{\sqrt{6}}{3|g(t)|} \sum_{k=1}^{\infty}\left|\varphi_{2 k}^{\prime \prime \prime}\right| \\
& +\left(\frac{\sqrt{6}+\sqrt{3}}{3|g(t)|}\right)\|b(x, t)\|_{L_{2}(D)}\left\|u^{(N)}(t)\right\|_{B} \\
& +\left(\frac{\sqrt{6}+\sqrt{3}}{3|g(t)|}\right) M .
\end{aligned}
$$


We deduce that $p^{(N)}(t) \in \mathbf{B}$.

Now we prove that the iterations $u^{(N+1)}(t)$ and $p^{(N+1)}(t)$ converge in $\mathbf{B}$ as $N \rightarrow \infty$.

$$
\begin{aligned}
& u_{0}^{(1)}(t)-u_{0}^{(0)}(t) \\
& =\int_{0}^{t} \int_{0}^{1}\left[f\left(\xi, \tau, u^{(0)}(\xi, \tau)\right)-f(\xi, \tau, 0)\right] \xi d \xi d \tau+\int_{0}^{t} \int_{0}^{1} f(\xi, \tau, 0) \xi d \xi d \tau, \\
& u_{2 k}^{(1)}(t)-u_{2 k}^{(0)}(t) \\
& =\int_{0}^{t} \int_{0}^{1}\left[f\left(\xi, \tau, u^{(0)}(\xi, \tau)\right)-f(\xi, \tau, 0)\right] e^{-(2 \pi k)^{2}(t-\tau)} \sin 2 \pi k \xi d \xi d \tau \\
& \quad+\int_{0}^{t} \int_{0}^{1} f(\xi, \tau, 0) e^{-(2 \pi k)^{2}(t-\tau)} \sin 2 \pi k \xi d \xi d \tau, \\
& u_{2 k-1}^{(1)}(t)-u_{2 k-1}^{(0)}(t) \\
& =\int_{0}^{t} \int_{0}^{1}\left[f\left(\xi, \tau, u^{(0)}(\xi, \tau)\right)-f(\xi, \tau, 0)\right] e^{-(2 \pi k)^{2}(t-\tau)} \xi \cos 2 \pi k \xi d \xi d \tau \\
& \quad+\int_{0}^{t} \int_{0}^{1} f(\xi, \tau, 0) e^{-(2 \pi k)^{2}(t-\tau)} \xi \cos 2 \pi k \xi d \xi d \tau \\
& \quad-4 \pi k \int_{0}^{t} \int_{0}^{1}\left[f\left(\xi, \tau, u^{(0)}(\xi, \tau)\right)-f(\xi, \tau, 0)\right](t-\tau) e^{-(2 \pi k)^{2}(t-\tau)} \sin 2 \pi k \xi d \xi d \tau \\
& \quad-4 \pi k \int_{0}^{t} \int_{0}^{1}(t-\tau) f(\xi, \tau, 0) e^{-(2 \pi k)^{2}(t-\tau)} \sin 2 \pi k \xi d \xi d \tau .
\end{aligned}
$$

Applying Cauchy's inequality, Hölder's inequality, the Lipschitz condition and Bessel's inequality to the last equation, we obtain

$$
\begin{aligned}
& \left|u^{(1)}(t)-u^{(0)}(t)\right| \leq\left(2 \sqrt{T}+\frac{2 \sqrt{3}}{3}+4 \sqrt{2} T\right)\left(\|b(x, t)\|_{L_{2}(D)}\left\|u^{(0)}(t)\right\|_{B}\right) \\
& +\left(2 \sqrt{T}+\frac{2 \sqrt{3}}{3}+4 \sqrt{2} T\right)\|f(x, t, 0)\|_{L_{2}(D)} \\
& K=\left(2 \sqrt{T}+\frac{2 \sqrt{3}}{3}+4 \sqrt{2} T\right)\left(\|b(x, t)\|_{L_{2}(D)}\left\|u^{(0)}(t)\right\|_{B}\right) \\
& +\left(2 \sqrt{T}+\frac{2 \sqrt{3}}{3}+4 \sqrt{2} T\right)\|f(x, t, 0)\|_{L_{2}(D)}, \\
& u_{0}^{(2)}(t)-u_{0}^{(1)}(t) \\
& =\int_{0}^{t} \int_{0}^{1}\left[f\left(\xi, \tau, u^{(1)}(\xi, \tau)\right)-f\left(\xi, \tau, u^{(0)}(\xi, \tau)\right)\right] \xi e^{-\int_{\tau}^{t} p^{(1)}(s) d s} d \xi d \tau \\
& +\int_{0}^{t} \int_{0}^{1} f\left(\xi, \tau, u^{(0)}(\xi, \tau)\right) \xi\left[e^{-\int_{\tau}^{t} p^{(1)}(s) d s}-e^{-\int_{\tau}^{t} p^{(0)}(s) d s}\right] d \xi d \tau, \\
& u_{2 k}^{(2)}(t)-u_{2 k}^{(1)}(t) \\
& =\int_{0}^{t} \int_{0}^{1}\left[f\left(\xi, \tau, u^{(1)}(\xi, \tau)\right)-f\left(\xi, \tau, u^{(0)}(\xi, \tau)\right)\right] e^{-(2 \pi k)^{2}(t-\tau)} e^{-\int_{\tau}^{t} p^{(1)}(s) d s} \sin 2 \pi k \xi d \xi d \tau \\
& +\int_{0}^{t} \int_{0}^{1} f\left(\xi, \tau, u^{(0)}(\xi, \tau)\right) \sin 2 k \xi e^{-(2 \pi k)^{2}(t-\tau)}\left[e^{-\int_{\tau}^{t} p^{(1)}(s) d s}-e^{-\int_{\tau}^{t} p^{(0)}(s) d s}\right] d \xi d \tau,
\end{aligned}
$$




$$
\begin{aligned}
u_{2 k-1}^{(2)}( & t)-u_{2 k-1}^{(1)}(t) \\
= & \int_{0}^{t} \int_{0}^{1}\left[f\left(\xi, \tau, u^{(1)}(\xi, \tau)\right)-f\left(\xi, \tau, u^{(0)}(\xi, \tau)\right)\right] \\
& \times e^{-(2 \pi k)^{2}(t-\tau)} e^{-\int_{\tau}^{t} p^{(1)}(s) d s} \xi \cos 2 \pi k \xi d \xi d \tau \\
& +\int_{0}^{t} \int_{0}^{1} f\left(\xi, \tau, u^{(0)}(\xi, \tau)\right) \xi \cos 2 \pi k \xi e^{-(2 \pi k)^{2}(t-\tau)} \\
& \times\left[e^{-\int_{\tau}^{t} p^{(1)}(s) d s}-e^{-\int_{\tau}^{t} p^{(0)}(s) d s}\right] d \xi d \tau \\
& -4 \pi k \int_{0}^{t} \int_{0}^{1}(t-\tau)\left[f\left(\xi, \tau, u^{(1)}(\xi, \tau)\right)-f\left(\xi, \tau, u^{(0)}(\xi, \tau)\right)\right] \\
& \times e^{-(2 \pi k)^{2}(t-\tau)} e^{-\int_{\tau}^{t} p^{(1)}(s) d s} \sin 2 \pi k \xi d \xi d \tau \\
& -4 \pi k \int_{0}^{t} \int_{0}^{1}(t-\tau) f\left(\xi, \tau, u^{(0)}(\xi, \tau)\right) \\
& \times e^{-(2 \pi k)^{2}(t-\tau)}\left[e^{-\int_{\tau}^{t} p^{(1)}(s) d s}-e^{-\int_{\tau}^{t} p^{(0)}(s) d s}\right] \sin 2 \pi k \xi d \xi d \tau .
\end{aligned}
$$

Applying Cauchy's inequality, Hölder's inequality, the Lipschitz condition and Bessel's inequality to the last equation, we obtain

$$
\begin{aligned}
\left|u^{(2)}(t)-u^{(1)}(t)\right| \leq & \left(2 \sqrt{T}+\frac{2 \sqrt{3}}{3}+4 \sqrt{2} T\right)\left(\|b(x, t)\|_{L_{2}(D)}\left|u^{(1)}-u^{(0)}\right|\right) \\
& +\left(2 \sqrt{T}+\frac{2 \sqrt{3}}{3}+4 \sqrt{2} T\right)|T|\|f(x, t, 0)\|_{L_{2}(D)}\left|p^{(1)}-p^{(0)}\right|, \\
p^{(1)}-p^{(0)}= & \frac{1}{g(t)} \sum_{k=1}^{\infty}\left(8 \pi k \int_{0}^{1}\left[f\left(\xi, \tau, u^{(1)}\right)-f\left(\xi, \tau, u^{(0)}\right)\right] \sin 2 \pi k \xi d \xi\right) \\
& -\frac{1}{g(t)} \sum_{k=1}^{\infty} 4(2 \pi k)^{2} \varphi_{2 k} e^{-(2 \pi k)^{2} t}\left[e^{-\int_{0}^{t} p^{(1)}(s) d s}-e^{\left.-\int_{0}^{t} p^{(0)}(s) d s\right]}\right. \\
& -\frac{1}{g(t)} \sum_{k=1}^{\infty} 4(2 \pi k)^{2} \int_{0}^{t} \int_{0}^{1}\left[f\left(\xi, \tau, u^{(1)}\right)-f\left(\xi, \tau, u^{(0)}\right)\right] \\
& \times \sin 2 \pi k \xi e^{-(2 \pi k)^{2}(t-\tau)} e^{-\int_{0}^{t} p^{(1)}(s) d s} d \xi d \tau \\
& -\frac{1}{g(t)} \sum_{k=1}^{\infty} 4(2 \pi k)^{2} \int_{0}^{t} \int_{0}^{1} f\left(\xi, \tau, u^{(0)}\right) \\
& \times \sin 2 \pi k \xi e^{-(2 \pi k)^{2}(t-\tau)}\left[e^{-\int_{0}^{t} p^{(1)}(s) d s}-e^{\left.-\int_{0}^{t} p^{(0)}(s) d s\right] d \xi d \tau .}\right.
\end{aligned}
$$

Applying Cauchy's inequality, Hölder's inequality, the Lipschitz condition and Bessel's inequality to the last equation, we obtain

$$
\begin{aligned}
\left|p^{(1)}-p^{(0)}\right| \leq & \left(\frac{\sqrt{6}+\sqrt{3}}{3|g(t)|}\right)\|b(x, t)\|_{L_{2}(D)}\left|u^{(1)}-u^{(0)}\right| \\
& +\left(\frac{\sqrt{3}}{3|g(t)|} M+\frac{\sqrt{6}|T|}{3|g(t)|} \sum_{k=1}^{\infty}\left|\varphi_{2 k-1}^{\prime \prime \prime}\right|\right)\left|p^{(1)}-p^{(0)}\right|
\end{aligned}
$$




$$
\begin{aligned}
& A=\left(\frac{\sqrt{6}+\sqrt{3}}{3|g(t)|}\right), \quad B=\left(\frac{\sqrt{3}}{3|g(t)|} M+\frac{\sqrt{6} T}{3|g(t)|} \sum_{k=1}^{\infty}\left|\varphi_{2 k-1}^{\prime \prime \prime}\right|\right), \quad B<1, \\
& \left|p^{(1)}-p^{(0)}\right| \leq \frac{A}{1-B}\|b(x, t)\|_{L_{2}(D)}\left|u^{(1)}-u^{(0)}\right|, \\
& \left|u^{(2)}(t)-u^{(1)}(t)\right| \leq\left(2 \sqrt{T}+\frac{2 \sqrt{3}}{3}+4 \sqrt{2} T\right)\left(\|b(x, t)\|_{L_{2}(D)}\left|u^{(1)}-u^{(0)}\right|\right) \\
& +\left(2 \sqrt{T}+\frac{2 \sqrt{3}}{3}+4 \sqrt{2} T\right) T \frac{M A}{1-B}\|b(x, t)\|_{L_{2}(D)}\left|u^{(1)}-u^{(0)}\right|, \\
& \left|u^{(2)}(t)-u^{(1)}(t)\right| \leq\left(\left(2 \sqrt{T}+\frac{2 \sqrt{3}}{3}+4 \sqrt{2} T\right)\left(1+\frac{T M A}{1-B}\right)\right)\|b(x, t)\|_{L_{2}(D)} K .
\end{aligned}
$$

For $N$, we have

$$
\begin{aligned}
& \left|p^{(N+1)}-p^{(N)}\right| \leq \frac{A}{1-B}\|b(x, t)\|_{L_{2}(D)}\left|u^{(N+1)}-u^{(N)}\right|, \\
& \left|u^{(N+1)}(t)-u^{(N)}(t)\right| \\
& \quad \leq \frac{1}{\sqrt{N !}}\left(\left(2 \sqrt{T}+\frac{2 \sqrt{3}}{3}+4 \sqrt{2} T\right)\left(1+\frac{M A T}{1-B}\right)\right)^{N}\|b(x, t)\|_{L_{2}(D)} K .
\end{aligned}
$$

It is easy to see that $u^{(N+1)} \rightarrow u^{(N)}, N \rightarrow \infty$, then $p^{(N+1)} \rightarrow p^{(N)}, N \rightarrow \infty$.

Therefore $u^{(N+1)}(t)$ and $p^{(N+1)}(t)$ converge in $\mathbf{B}$.

Now let us show that there exist $u$ and $p$ such that

$$
\lim _{N \rightarrow \infty} u^{(N+1)}(t)=u(t), \quad \lim _{N \rightarrow \infty} p^{(N+1)}(t)=p(t) .
$$

In the same way, we obtain

$$
\begin{aligned}
&\left|u(t)-u^{(N+1)}(t)\right| \leq\left(2 \sqrt{T}+\frac{2 \sqrt{3}}{3}+4 \sqrt{2} T\right)\|b(x, t)\|_{L_{2}(D)}\left\|u(\tau)-u^{(N+1)}(\tau)\right\|_{B} \\
&+\left(2 \sqrt{T}+\frac{2 \sqrt{3}}{3}+4 \sqrt{2} T\right)\|b(x, t)\|_{L_{2}(D)}\left\|u^{(N+1)}(\tau)-u^{(N)}(\tau)\right\|_{B} \\
&+\left(2 \sqrt{T}+\frac{2 \sqrt{3}}{3}+4 \sqrt{2} T\right)|T|\left|p(\tau)-p^{(N)}(\tau)\right|\|f(x, t, u)\|, \\
&\left|p-p^{(N)}\right| \leq \frac{A}{1-B}\left(\int_{0}^{t} \int_{0}^{1} b^{2}(\xi, \tau)\left|u(\tau)-u^{(N+1)}(\tau)\right|^{2} d \xi d \tau\right)^{\frac{1}{2}} \\
&+\frac{A}{1-B}\left(\int_{0}^{t} \int_{0}^{1} b^{2}(\xi, \tau)\left|u^{(N+1)}(\tau)-u^{(N)}(\tau)\right|^{2} d \xi d \tau\right)^{\frac{1}{2}} .
\end{aligned}
$$

Applying Gronwall's inequality to (10) and using (9) and (11), we have

$$
\begin{aligned}
\left\|u(t)-u^{(N+1)}(t)\right\|_{B} \leq & 2\left[\frac{K}{\sqrt{N !}} D^{2} E^{2}\|b(x, t)\|_{L_{2}(D)}\right]^{2} \\
& \times \exp 2\left(D+D^{2}|T| \frac{M A}{1-B}\right)^{2}\|b(x, t)\|_{L_{2}(D)}^{2}
\end{aligned}
$$


Here

$$
D=\left(2 \sqrt{T}+\frac{2 \sqrt{3}}{3}+4 \sqrt{2} T\right), \quad E=\left\{\left(2 \sqrt{T}+\frac{2 \sqrt{3}}{3}+4 \sqrt{2} T\right)\left(1+T \frac{M A}{1-B}\right)\right\}^{N} .
$$

Then $N \rightarrow \infty$, we obtain $u^{(N+1)} \rightarrow u$. Hence $p^{(N+1)} \rightarrow p$.

For the uniqueness, we assume that problem (1)-(4) has two solutions $(p, u),(q, v)$. Applying Cauchy's inequality, Hölder's inequality, the Lipschitz condition and Bessel's inequality to $|u(t)-v(t)|$ and $|p(t)-q(t)|$, we obtain

$$
\begin{aligned}
|u(t)-v(t)| \leq & \left(\|\varphi\|+\left(2 \sqrt{T}+\frac{2 \sqrt{3}}{3}+4 \sqrt{2} T\right) M\right) T|p(t)-q(t)| \\
& +\left(2 \sqrt{T}+\frac{2 \sqrt{3}}{3}+4 \sqrt{2} T\right)\left(\int_{0}^{t} \int_{0}^{\pi} b^{2}(\xi, \tau)|u(\tau)-v(\tau)|^{2} d \xi d \tau\right)^{\frac{1}{2}}, \\
|p(t)-q(t)| \leq & \frac{A}{1-B}\left(\int_{0}^{t} \int_{0}^{1} b^{2}(\xi, \tau)|u(\tau)-v(\tau)|^{2} d \xi d \tau\right)^{\frac{1}{2}}, \\
|u(t)-v(t)| \leq & {\left[\left(\|\varphi\|+\left(2 \sqrt{T}+\frac{2 \sqrt{3}}{3}+4 \sqrt{2} T\right) M\right)|T| \frac{A}{1-B}\right.} \\
& \left.+\left(2 \sqrt{T}+\frac{2 \sqrt{3}}{3}+4 \sqrt{2} T\right)\right] \\
& \times\left(\int_{0}^{t} \int_{0}^{1} b^{2}(\xi, \tau)|u(\tau)-v(\tau)|^{2} d \xi d \tau\right)^{\frac{1}{2}} .
\end{aligned}
$$

Applying Gronwall's inequality to (13), we have $u(t)=v(t)$. Hence $p(t)=q(t)$.

The theorem is proved.

\section{Continuous dependence of $(p, u)$ upon the data}

Theorem 4 Under assumptions (A1)-(A3), the solution $(p, u)$ of problem (1)-(4) depends continuously upon the data $\varphi, g$.

Proof Let $\Phi=\{\varphi, g, f\}$ and $\bar{\Phi}=\{\bar{\varphi}, \bar{g}, f\}$ be two sets of the data, which satisfy assumptions (A1)-(A3). Suppose that there exist positive constants $M_{i}, i=0,1,2$, such that

$$
\begin{aligned}
& 0<M_{0} \leq|g|, \quad 0<M_{0} \leq|\bar{g}|, \quad\|g\|_{C^{1}[0, T]} \leq M_{1}, \quad\|\bar{g}\|_{C^{1}[0, T]} \leq M_{1}, \\
& \|\varphi\|_{C^{3}[0, \pi]} \leq M_{2}, \quad\|\bar{\varphi}\|_{C^{3}[0, \pi]} \leq M_{2} .
\end{aligned}
$$

Let us denote $\|\Phi\|=\left(\|g\|_{C^{1}[0, T]}+\|\varphi\|_{C^{3}[0, \pi]}+\|f\|_{C^{3,0}(\bar{D})}\right)$. Let $(p, u)$ and $(\bar{p}, \bar{u})$ be the solutions of inverse problem (1)-(4) corresponding to the data $\Phi=\{\varphi, g, f\}$ and $\bar{\Phi}=\{\bar{\varphi}, \bar{g}, f\}$, respectively. According to (5),

$$
\begin{aligned}
u-\bar{u}= & 2\left(\varphi_{0}-\overline{\varphi_{0}}\right) e^{-\int_{0}^{t} \bar{p}(s) d s}+\varphi_{0}\left(e^{-\int_{0}^{t} p(s) d s}-e^{-\int_{0}^{t} \bar{p}(s) d s}\right) \\
& +2 \int_{0}^{t} \int_{0}^{1}[f(\xi, \tau, u(\xi, \tau))-f(\xi, \tau, \bar{u}(\xi, \tau))] \xi e^{-\int_{\tau}^{t} \bar{p}(s) d s} d \xi d \tau
\end{aligned}
$$




$$
\begin{aligned}
& +2 \int_{0}^{t} \int_{0}^{1} f(\xi, \tau, u(\xi, \tau))\left[e^{-\int_{\tau}^{t} p(s) d s}-e^{-\int_{\tau}^{t} \bar{p}(s) d s}\right] \\
& +4 \sum_{k=1}^{\infty}(1-x) \sin 2 \pi k \xi\left(\varphi_{2 k}-\overline{\varphi_{2 k}}\right) e^{-(2 \pi k)^{2} t}\left[e^{-\int_{\tau}^{t} p(s) d s}-e^{-\int_{\tau}^{t} \bar{p}(s) d s}\right] \\
& +4 \sum_{k=1}^{\infty}(1-x) \sin 2 \pi k \xi \varphi_{2 k} e^{-(2 \pi k)^{2} t} e^{-\int_{\tau}^{t} \bar{p}(s) d s} \\
& +4 \sum_{k=1}^{\infty} \cos 2 \pi k \xi\left(\varphi_{2 k-1}-\overline{\varphi_{2 k-1}}\right) e^{-(2 \pi k)^{2} t}\left[e^{-\int_{\tau}^{t} p(s) d s}-e^{-\int_{\tau}^{t} \bar{p}(s) d s}\right] \\
& +4 \sum_{k=1}^{\infty} \cos 2 \pi k \xi \varphi_{2 k-1} e^{-(2 k)^{2} t} e^{-\int_{\tau}^{t} \bar{p}(s) d s} \\
& -16 \pi \sum_{k=1}^{\infty} k t \cos 2 \pi k \xi\left(\varphi_{2 k}-\overline{\varphi_{2 k}}\right) e^{-(2 \pi k)^{2} t}\left[e^{-\int_{\tau}^{t} p(s) d s}-e^{-\int_{\tau}^{t} \bar{p}(s) d s}\right] \\
& -16 \pi \sum_{k=1}^{\infty} k t \cos 2 \pi k \xi \varphi_{2 k} e^{-(2 \pi k)^{2} t} e^{-\int_{\tau}^{t} \bar{p}(s) d s} \\
& +4 \sum_{k=1}^{\infty} \int_{0}^{t} \int_{0}^{1}[f(\xi, \tau, u(\xi, \tau))-f(\xi, \tau, \bar{u}(\xi, \tau))] \\
& \times \sin 2 \pi k \xi e^{-(2 \pi k)^{2}(t-\tau)-\int_{\tau}^{t} \bar{p}(s) d s} d \xi d \tau \\
& +4 \sum_{k=1}^{\infty} \int_{0}^{t} \int_{0}^{1} f(\xi, \tau, u(\xi, \tau)) \\
& \times \sin 2 \pi k \xi e^{-(2 \pi k)^{2}(t-\tau)}\left[e^{-\int_{\tau}^{t} p(s) d s}-e^{-\int_{\tau}^{t} \bar{p}(s) d s}\right] d \xi d \tau \\
& +4 \sum_{k=1}^{\infty} \int_{0}^{t} \int_{0}^{1}[f(\xi, \tau, u(\xi, \tau))-f(\xi, \tau, \bar{u}(\xi, \tau))] \\
& \times \xi \cos 2 \pi k \xi e^{-(2 \pi k)^{2}(t-\tau)-\int_{\tau}^{t} \bar{p}(s) d s} d \xi d \tau \\
& +4 \sum_{k=1}^{\infty} \int_{0}^{t} \int_{0}^{1} f(\xi, \tau, u(\xi, \tau)) \\
& \times \xi \cos 2 \pi k \xi e^{-(2 \pi k)^{2}(t-\tau)}\left[e^{-\int_{\tau}^{t} p(s) d s}-e^{-\int_{\tau}^{t} \bar{p}(s) d s}\right] d \xi d \tau \\
& -16 \pi \sum_{k=1}^{\infty} k \int_{0}^{t} \int_{0}^{1}[f(\xi, \tau, u(\xi, \tau))-f(\xi, \tau, \bar{u}(\xi, \tau))] \\
& \times(t-\tau) \sin 2 \pi k \xi e^{-(2 \pi k)^{2}(t-\tau)-\int_{\tau}^{t} \bar{p}(s) d s} d \xi d \tau \\
& -16 \pi \sum_{k=1}^{\infty} k \int_{0}^{t} \int_{0}^{1} f(\xi, \tau, u(\xi, \tau)) \\
& \times(t-\tau) \sin 2 \pi k \xi e^{-(2 \pi k)^{2}(t-\tau)}\left[e^{-\int_{\tau}^{t} p(s) d s}-e^{-\int_{\tau}^{t} \bar{p}(s) d s}\right] d \xi d \tau, \\
& |u-\bar{u}| \leq\left(\left(2 \sqrt{T}+\frac{2 \sqrt{3}}{3}+4 \sqrt{2} T\right)+\frac{8 \pi^{2} \sqrt{6}}{3} \sum_{k=0}^{\infty}\left|\varphi_{2 k}^{\prime \prime \prime}\right|+4 \sum_{k=0}^{\infty}\left(\left|\varphi_{2 k}\right|+\left|\varphi_{2 k-1}\right|\right)\right)
\end{aligned}
$$




$$
\begin{aligned}
& \times\|p-\bar{p}\|_{C[0, T]}+\left(1+\frac{2 \sqrt{6}}{3} T\right)\|\varphi-\bar{\varphi}\|_{C^{3}[0,1]} \\
& +\left(2 \sqrt{T}+\frac{2 \sqrt{3}}{3}+4 \sqrt{2} T\right)\left(\int_{0}^{t} \int_{0}^{1} b^{2}(\xi, \tau)|u(\tau)-\bar{u}(\tau)|^{2} d \xi d \tau\right)^{\frac{1}{2}} .
\end{aligned}
$$

Now, let us estimate the difference $p-\bar{p}$ as follows:

$$
\begin{aligned}
p-\bar{p}= & \left(\frac{-g^{\prime}(t)}{g(t)}+\frac{\overline{g^{\prime}(t)}}{\overline{g(t)}}\right) \\
& +\frac{1}{g(t)} \sum_{k=1}^{\infty} 8 \pi k \int_{0}^{1} f(\xi, \tau, u) \sin 2 \pi k \xi d \xi \\
& -\frac{1}{\bar{g}(t)} \sum_{k=1}^{\infty} 8 \pi k \int_{0}^{1} f(\xi, \tau, \bar{u}) \sin 2 \pi k \xi d \xi \\
& -4 \frac{1}{g(t)} \sum_{k=1}^{\infty}(2 \pi k)^{2} \varphi_{2 k} e^{-(2 \pi k)^{2} t} e^{-\int_{0}^{t} p(s) d s} \\
& +4 \frac{1}{\bar{g}(t)} \sum_{k=1}^{\infty}(2 \pi k)^{2} \overline{\varphi_{2 k}} e^{-(2 \pi k)^{2} t} e^{-\int_{0}^{t} \overline{p(s)} d s} \\
& -4 \frac{1}{g(t)} \sum_{k=1}^{\infty}(2 \pi k)^{2} \int_{0}^{t} \int_{0}^{1} f(\xi, \tau, u) \sin 2 \pi k \xi e^{-(2 \pi k)^{2}(t-\tau)-\int_{\tau}^{t} p(s) d s} d \xi d \tau \\
& -4 \frac{1}{\bar{g}(t)} \sum_{k=1}^{\infty}(2 \pi k)^{2} \int_{0}^{t} \int_{0}^{1} f(\xi, \tau, \bar{u}) \sin 2 \pi k \xi e^{-(2 \pi k)^{2}(t-\tau)-\int_{\tau}^{t} \overline{p(s)} d s} d \xi d \tau, \\
\|p-\bar{p}\|_{C[0, T]} \leq M_{3}\|g-\bar{g}\|_{C^{1}[0, T]}+M_{4}\|\varphi-\bar{\varphi}\|_{C^{3}[0,1]} & +M_{5} T\|p-\bar{p}\|_{C[0, T]}+M_{6}\left(\int_{0}^{t} \int_{0}^{\pi} b^{2}(\xi, \tau)|u(\tau)-\bar{u}(\tau)|^{2} d \xi d \tau\right)^{\frac{1}{2}},
\end{aligned}
$$

where $M_{k}, k=3,4,5,6$ are constants that are determined by $M_{0}, M_{1}$ and $M_{2}$. Then we obtain $M_{7}=1-T M_{5}, M_{8}=\max \left\{M_{3}, M_{4}, M_{6}\right\}$. The inequality $M_{5} T<1$ holds for small $T$. Finally, we obtain

$$
\begin{aligned}
\|p-\bar{p}\|_{C[0, T]} \leq & M_{9}\left(\|E-\bar{E}\|_{C^{1}[0, T]}+\|\varphi-\bar{\varphi}\|_{C^{3}[0,1]}\right. \\
& \left.+\left(\int_{0}^{t} \int_{0}^{\pi} b^{2}(\xi, \tau)|u(\tau)-\bar{u}(\tau)|^{2} d \xi d \tau\right)^{\frac{1}{2}}\right),
\end{aligned}
$$

where $M_{9}=\frac{M_{8}}{M_{7}}$.

If we take this estimation in (14)

$$
\begin{aligned}
& |u-\bar{u}| \leq M_{12}\|\Phi-\bar{\Phi}\|+M_{13}\left(\int_{0}^{t} \int_{0}^{1} b^{2}(\xi, \tau)|u(\tau)-\bar{u}(\tau)|^{2} d \xi d \tau\right)^{\frac{1}{2}}, \\
& |u-\bar{u}| \leq M_{12}\|\Phi-\bar{\Phi}\|+M_{13}\left(\int_{0}^{t} \int_{0}^{1} b^{2}(\xi, \tau)|u(\tau)-\bar{u}(\tau)|^{2} d \xi d \tau\right)^{\frac{1}{2}}, \\
& |u-\bar{u}|^{2} \leq 2 M_{12}^{2}\|\Phi-\bar{\Phi}\|^{2}+2 M_{13}^{2}\left(\int_{0}^{t} \int_{0}^{\pi} b^{2}(\xi, \tau)|u(\tau)-\bar{u}(\tau)|^{2} d \xi d \tau\right),
\end{aligned}
$$


applying Gronwall's inequality, we obtain

$$
|u-\bar{u}|^{2} \leq 2 M_{12}^{2}\|\Phi-\bar{\Phi}\|^{2} x \exp 2 M_{13}^{2}\left(\int_{0}^{t} \int_{0}^{1} b^{2}(\xi, \tau) d \xi d \tau\right)
$$

taking the maximum of the inequality

$$
\|u-\bar{u}\|_{B}^{2} \leq 2 M_{12}^{2}\|\Phi-\bar{\Phi}\|^{2} x \exp 2 M_{13}^{2}\left(\int_{0}^{t} \int_{0}^{1} b^{2}(\xi, \tau) d \xi d \tau\right)
$$

For $\Phi \rightarrow \bar{\Phi}$, then $u \rightarrow \bar{u}$. Hence $p \rightarrow \bar{p}$.

\section{Numerical procedure for nonlinear problem (1)-(4)}

We construct an iteration algorithm for the linearization of problem (1)-(4) as follows:

$$
\begin{aligned}
& \frac{\partial u^{(n)}}{\partial t}=\frac{\partial^{2} u^{(n)}}{\partial x^{2}}-p(t) u^{(n)}+f\left(x, t, u^{(n-1)}\right), \quad(x, t) \in D, \\
& u^{(n)}(0, t)=u^{(n)}(1, t), \quad t \in[0, T], \\
& u_{x}^{(n)}(1, t)=0, \quad t \in[0, T], \\
& u^{(n)}(x, 0)=\varphi(x), \quad x \in[0,1] .
\end{aligned}
$$

Let $u^{(n)}(x, t)=v(x, t)$ and $f\left(x, t, u^{(n-1)}\right)=\widetilde{f}(x, t)$. Then problem (15)-(18) can be written as a linear problem:

$$
\begin{aligned}
& \frac{\partial v}{\partial t}=\frac{\partial^{2} v}{\partial x^{2}}-p(t) v(x, t)+\widetilde{f}(x, t), \quad(x, t) \in D, \\
& v(0, t)=v(1, t), \quad t \in[0, T], \\
& v_{x}(1, t)=0, \quad t \in[0, T], \\
& v(x, 0)=\varphi(x), \quad x \in[0,1] .
\end{aligned}
$$

We use the finite difference method to solve (19)-(22) with a predictor-corrector type approach which was explained in [9].

We subdivide the intervals $[0,1]$ and $[0, T]$ into $N_{x}$ and $N_{t}$ subintervals of equal lengths $h=\frac{1}{N_{x}}$ and $\tau=\frac{T}{N_{t}}$, respectively. Then we add two lines $x=0$ and $x=\left(N_{x}+1\right) h$ to generate the fictitious points needed for dealing with the boundary conditions. We choose the implicit scheme, which is absolutely stable and has second-order accuracy in $h$ and first-order accuracy in $\tau$ [10]. The implicit scheme for (1)-(4) is as follows:

$$
\begin{aligned}
& \frac{1}{\tau}\left(v_{i}^{j+1}-v_{i}^{j}\right)=\frac{1}{h^{2}}\left(v_{i-1}^{j+1}-2 v_{i}^{j+1}+v_{i+1}^{j+1}\right)-p^{j+1} v_{i}^{j+1}+\widetilde{f}_{i}^{j+1}, \\
& v_{i}^{0}=\phi_{i}, \\
& v_{0}^{j}=v_{N_{x}+1}^{j}, \\
& v_{N_{x}-1}^{j}=v_{N_{x}+1}^{j},
\end{aligned}
$$


where $1 \leq i \leq N_{x}$ and $1 \leq j \leq N_{t}$ are the indices for the spatial and time steps, respectively, $v_{i}^{j}=v\left(x_{i}, t_{j}\right), \phi_{i}=\varphi\left(x_{i}\right), \widetilde{f}_{i}^{j}=\widetilde{f}\left(x_{i}, t_{j}\right), x_{i}=i h, t_{j}=j \tau$. At $t=0$ level, adjustment should be made according to the initial condition and the compatibility requirements.

Now, let us construct the predicting-correcting mechanism. First, differentiating equation (1) with respect to $x$ and using (3) and (4), we obtain

$$
p(t)=\frac{-g^{\prime}(t)+\tilde{f}_{x}(0, t) d x+v_{x x x}(0, t)}{g(t)} .
$$

The finite difference approximation of (27) is

$$
p^{j}=\frac{-\left(\left(g^{j+1}-g^{j}\right) / \tau\right)+\left(\widetilde{f}_{2}^{j}-\widetilde{f}_{1}^{j}\right) / \tau+\left(-v_{0}^{j}+3 v_{1}^{j}-3 v_{2}^{j}+v_{3}^{j}\right) / h^{3}}{g^{j}},
$$

where $g^{j}=g\left(t_{j}\right), j=0,1, \ldots, N_{t}$.

$$
\text { For } j=0 \text {, }
$$

$$
p^{0}=\frac{-\left(\left(g^{1}-g^{0}\right) / \tau\right)+\left(\widetilde{f}_{2}^{0}-\widetilde{f}_{1}^{0}\right) / \tau+\left(-\phi_{0}+3 \phi 1-3 \phi_{2}+\phi_{3}\right) / h^{3}}{g^{0}},
$$

and the values of $\phi_{i}$ allow us to start our computation. We denote the values of $p^{j}, v_{i}^{j}$ at the $s$ th iteration step $p^{j(s)}, v_{i}^{j(s)}$, respectively. In numerical computation, since the time step is very small, we can take $p^{j+1(0)}=p^{j}, v_{i}^{j+1(0)}=v_{i}^{j}, j=0,1,2, \ldots, N_{t}, i=1,2, \ldots, N_{x}$. At each $(s+1)$ th iteration step, we first determine $p^{j+1(s+1)}$ from the formula

$$
p^{j+1(s+1)}=\frac{-\left(\left(g^{j+2}-g^{j+1}\right) / \tau\right)+\left(\widetilde{f}_{2}^{j+1}-\widetilde{f}_{1}^{j+1}\right) / \tau+\left(-v_{0}^{j+1(s)}+3 v_{1}^{j+1(s)}-3 v_{2}^{j+1(s)}+v_{3}^{j+1(s)}\right) / h^{3}}{g^{j+1}} .
$$

Then from (15)-(18) we obtain

$$
\begin{aligned}
& \frac{1}{\tau}\left(v_{i}^{j+1(s+1)}-v_{i}^{j+1(s)}\right)=\frac{1}{h^{2}}\left(v_{i-1}^{j+1(s+1)}-2 v_{i}^{j+1(s+1)}+v_{i+1}^{j+1(s+1)}\right)-p^{j+1(s+1)} v_{i}^{j+1(s+1)}+F_{i}^{j+1}, \\
& v_{0}^{j+1(s)}=v_{N_{x}+1}^{j+1(s)}, \\
& v_{N_{x}-1}^{j+1(s)}=v_{N_{x}+1}^{j+1(s)} .
\end{aligned}
$$

The system of equations (28)-(30) can be solved by the Gauss elimination method and $v_{i}^{j+1(s+1)}$ is determined. If the difference of values between two iterations reaches the prescribed tolerance, the iteration is stopped, and we accept the corresponding values $p^{j+1(s+1)}$, $v_{i}^{j+1(s+1)}\left(i=1,2, \ldots, N_{x}\right)$ as $p^{j+1}, v_{i}^{j+1}\left(i=1,2, \ldots, N_{x}\right)$, at the $(j+1)$ th time step, respectively. By virtue of this iteration, we can move from level $j$ to level $j+1$.

\section{Numerical example}

Example 1 Consider inverse problem (1)-(4) with

$$
\begin{aligned}
& f(x, t, u)=4 \pi \cos (2 \pi x)+\left((2 \pi)^{2}+\exp (2 t)\right) u, \\
& \varphi(x)=(1-x) \sin (2 \pi x), \quad g(t)=2 \pi \exp (-t), \quad x \in[0,1], t \in[0, T] .
\end{aligned}
$$


It is easy to check that the analytical solution of this problem is

$$
\{p(t), u(x, t)\}=\{1+\exp (2 t),(1-x) \sin (2 \pi x) \exp (-t)\} .
$$

Let us apply the scheme which was explained in the previous section for the step sizes $h=0.05, \tau=0.05$.

In the case when $T=1$, the comparisons between the analytical solution (31) and the numerical finite difference solution are shown in Figures 1 and 2.

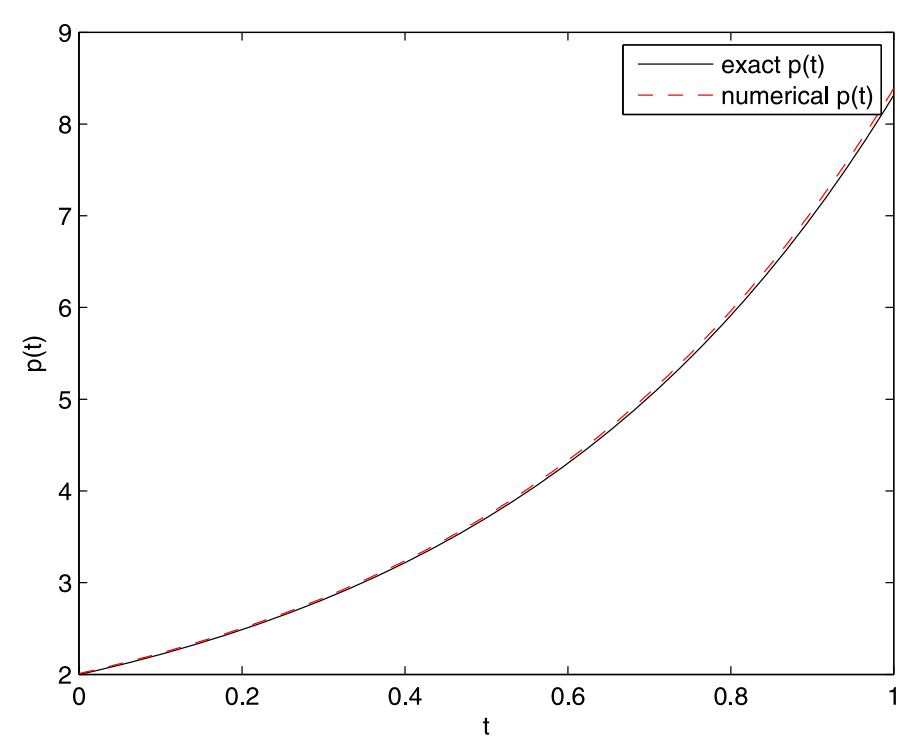

Figure 1 The analytical and numerical solutions of $p(t)$ when $T=1$. The analytical solution is shown with dashed line.

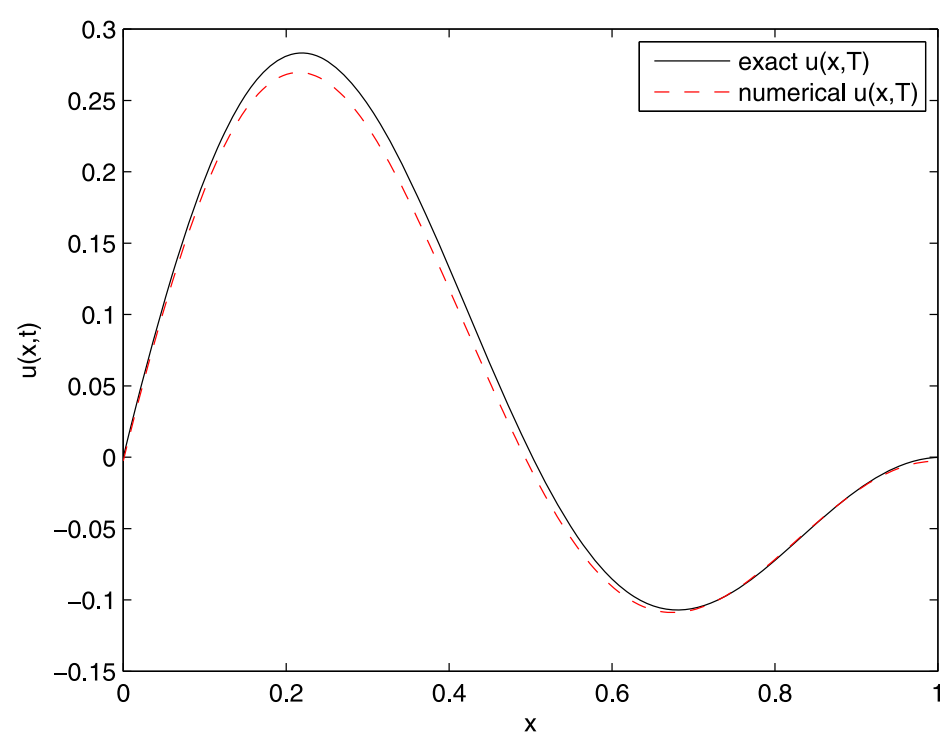

Figure 2 The analytical and numerical solutions of $u(x, t)$ at $T=1$. The analytical solution is shown with dashed line. 


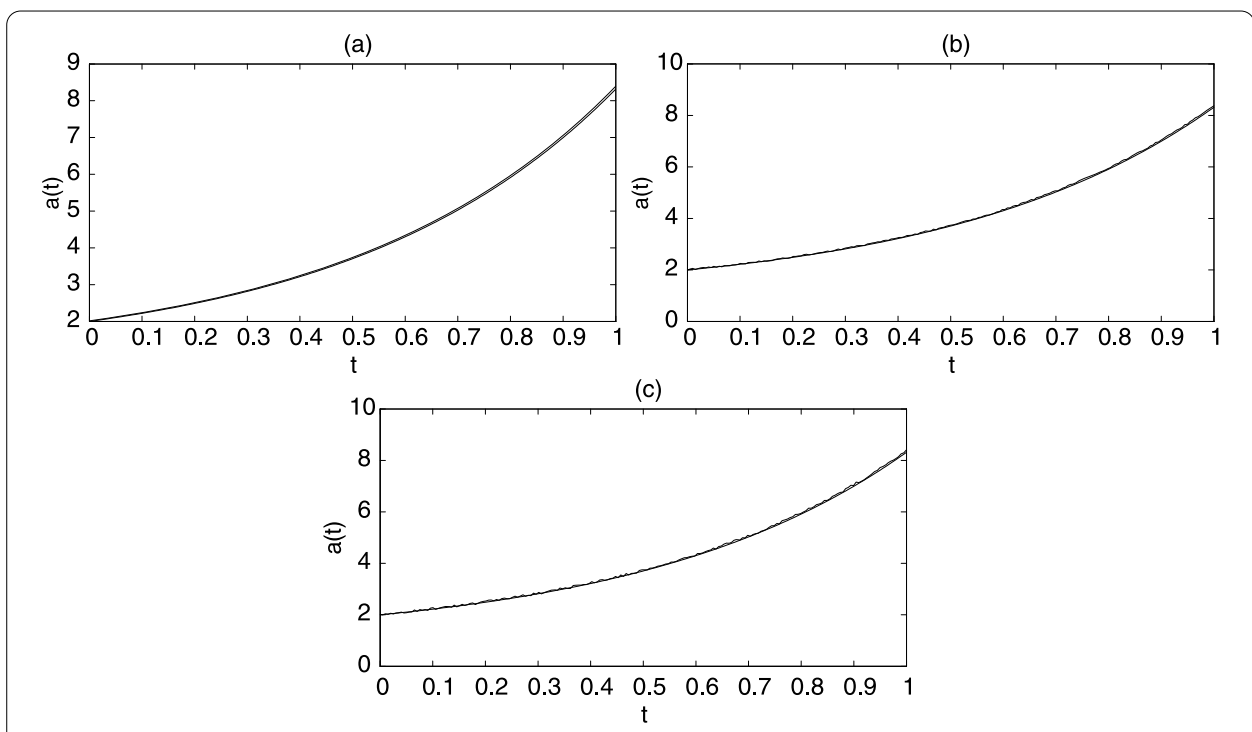

Figure 3 The numerical solutions of $p(t)$ (a) for $1 \%$ noisy data, (b) for $3 \%$ noisy data, (c) for $5 \%$ noisy data. In Figure 3(a)-(c) the analytical solution is shown with dashed line.

Next, we will illustrate the stability of the numerical solution with respect to the noisy overdetermination data (4) defined by the function

$$
g_{\gamma}(t)=g(t)(1+\gamma \theta)
$$

where $\gamma$ is the percentage of noise and $\theta$ are random variables generated from uniform distribution in the interval $[-1,1]$. Figure 3 shows the exact and the numerical solution of $p(t)$ when the input data (4) is contaminated by $\gamma=1 \%, \gamma=3 \%$ and $5 \%$ noise.

It is clear from these results that this method has shown to produce stable and reasonably accurate results for these examples. Numerical differentiation is used to compute the values of $g^{\prime}(t)$ and $v_{x x x}(0, t)$ in the formula $p(t)$. It is well known that numerical differentiation is slightly ill-posed and it can cause some numerical difficulties. One can apply the natural cubic spline function technique [11] to get still decent accuracy.

Competing interests

The authors declare that they have no competing interests.

Authors' contributions

FK conceived the study, participated in its design and coordination and prepared computing section. IB participated in the sequence alignment and achieved the estimation.

\section{Author details}

${ }^{1}$ Department of Management Information Systems, Kadir Has University, Istanbul, 34083, Turkey. ${ }^{2}$ Department of Mathematics, Kocaeli University, Kocaeli, 41380, Turkey.

Received: 7 June 2013 Accepted: 27 August 2013 Published: 30 Sep 2013

\section{References}

1. Cannon, J, Lin, Y: Determination of parameter $p(t)$ in Holder classes for some semilinear parabolic equations. Inverse Probl. 4, 595-606 (1988)

2. Pourgholia, R, Rostamiana, M, Emamjome, M: A numerical method for solving a nonlinear inverse parabolic problem. Inverse Probl. Sci. Eng. 18, 1151-1164 (2010)

3. Gatti, S: An existence result for an inverse problem for a quasilinear parabolic equation. Inverse Probl. 14, 53-65 (1998)

4. Namazov, G: Definition of the unknown coefficient of a parabolic equation with nonlocal boundary and complementary conditions. Trans. Acad. Sci. Azerb. Ser. Phys.-Tech. Math. Sci. 19, 113-117 (1999) 
5. Ismailov, M, Kanca, F: An inverse coefficient problem for a parabolic equation in the case of nonlocal boundary and overdetermination conditions. Math. Methods Appl. Sci. 34, 692-702 (2011)

6. Kanca, F, Ismailov, M: Inverse problem of finding the time-dependent coefficient of heat equation from integral overdetermination condition data. Inverse Probl. Sci. Eng. 20,463-476 (2012)

7. Nakhushev, AM: Equations of Mathematical Biology. Vysshaya Shkola, Moscow (1995)

8. Ionkin, N: Solution of a boundary-value problem in heat conduction with a nonclassical boundary condition. Differ. Equ. 13, 204-211 (1977)

9. Cannon, J, Lin, Y, Wang, S: Determination of source parameter in a parabolic equations. Meccanica 27, 85-94 (1992)

10. Samarskii, AA: The Theory of Difference Schemes. Dekker, New York (2001)

11. Atkinson, KE: Elementary Numerical Analysis. Wiley, New York (1985)

10.1186/1687-2770-2013-213

Cite this article as: Kanca and Baglan: An inverse coefficient problem for a quasilinear parabolic equation with nonlocal boundary conditions. Boundary Value Problems 2013, 2013:213

Submit your manuscript to a SpringerOpen ${ }^{\circ}$ journal and benefit from:

- Convenient online submission

- Rigorous peer review

- Immediate publication on acceptance

- Open access: articles freely available online

- High visibility within the field

- Retaining the copyright to your article 